\title{
Consideraciones para calcular el ratio precio-utilidad de la Bolsa de Valores de Lima: metodología y aplicaciones
}

\section{Price - Earnings Ratio for the Lima Stock Exchange: \\ Issues and Applications}

\author{
Javier Pereda 1
}

Received: December 26, 2011

Accepted: March 02, 2012

\section{Resumen}

En el presente trabajo se elabora una metodología para calcular el ratio precio-utilidad (PER) del Índice General de la Bolsa de Valores de Lima (IGBVL) en el periodo 1995-2011, dentro del enfoque planteado por Shiller (2005). A partir del PER calculado se estima la prima por riesgo del mercado de acciones (implicitequity premia), siguiendo la metodología planteada por Hayford y Malliaris (2004). Tanto el PER como la prima por riesgo estimadas se usan como indicadores para evaluar el desempeño del mercado de acciones en el periodo analizado. Los resultados muestran que los precios de las acciones respondieron básicamente a la evolución esperada de las utilidades de las empresas, incluso durante el periodo de auge de la bolsa de valores que precedió a la crisis financiera externa del 2008, sin evidencia de que haya existido una sobrevaluación en este mercado.

Palabras Claves: PER, ratio precio-utilidad, precio de activos, prima de rendimiento de acciones.

\begin{abstract}
In this paper we construct a methodology to calculate the price-earnings ratio (PER) of the General Index of the Lima Stock Exchange (IGBVL) for the period 1995-2011 following Shiller (2005). Results show that equity prices, in the analyzed period, basically responded to the expected evolution of earnings of the companies, even during the period of the equity prices boom that preceded the financial crisis of 2008. This conclusion is reinforced when we calculate, following Hayford y Malliaris (2004), the implicit equity premia expected for stock investors. We find high values of equity premia during the period of stock prices boom, which would justify the high PER values registered in that period.
\end{abstract}

Key Words: PER, price-earnings ratio, asset prices, equity premia.

1. Licenciado en Economía por la Pontificia Universidad Católica del Perú. M. Sc. en Economía, London School of Economics. $<$ javier.pereda@bcrp.gob.pe>. 


\section{INTRODUCCIÓN}

El comportamiento de los precios de los principales activos domésticos tiene especial relevancia para el manejo macroeconómico y monetario, y ha sido un elemento consustancial en las recientes crisis financieras. Carmassi, Gros y Micossi (2009) señalan dos indicadores cuantitativos que la literatura ha identificado como indicadores adelantados de crisis financieras: la excesiva expansión del crédito y el inusual incremento en los precios de los activos.

En este sentido, en la mayoría de países desarrollados se elaboran indicadores de precios del mercado inmobiliario, del mercado de acciones y del tipo de cambio, entre los principales activos. En el Perú, Banco Central de Reserva del Perú (BCRP) (2010) elabora un indicador para medir la evolución de los precios de los inmuebles ${ }^{1}$. Por su parte, Salas y Ferreyra (2006) y Winkelried y Rodríguez (2011) elaboran indicadores para medir el tipo de cambio real de equilibrio. En el caso de las acciones, no se cuenta con un indicador agregado que sirva para medir su evolución, lo que se torna relevante dada la creciente importancia de este mercado en los últimos 10 años.

El PER ${ }^{2}$ (por sus siglas en inglés Price Earning Ratio $)^{3}$ es uno de los indicadores más populares para medir la evolución de los precios de las acciones y es reportado por Bloomberg para las principales plazas bursátiles del mundo. En América Latina existe, por

1 El PER de los inmuebles (ratio precio de viviendas-costo de alquiler) se elabora a partir de información de precios (de oferta) de viviendas similares en 10 distritos de Lima (Jesús María, La Molina, Lince, Magdalena, Miraflores, Pueblo Libre, San Borja, San Isidro, San Miguel y Surco) y se le compara con valores referenciales proporcionados por la Global Property Guide (guía que no incluye al Perú). Con ello se determina si los precios de las viviendas están en sus valores "normales". Se diferencia de la metodología usada por S\&P/Case-Shiller para Estados Unidos, mercado que usa el método de comparación de ventas repetidas de una misma vivienda. Este método permite un ajuste mayor de características tales como: tamaño, ubicación, calidad, entre otras.

2 También se le conoce como ratio precio-utilidad (P/U) o ratio precio-ganancia $(\mathrm{P} / \mathrm{G})$.

3 Bloomberg publica la lista del PER de las principales bolsas mundiales en el ticker: WPE. ejemplo, información del PER para Chile, Colombia, México y Brasil.

En el presente trabajo se elabora una metodología para calcular el valor del PER agregado para el Perú del Índice General de la Bolsa de Valores de Lima (IGBVL). El PER estimado se ajusta siguiendo la metodología propuesta por Shiller (2005), y se usa para medir el desempeño del mercado de acciones en el periodo 1995-2011 con datos mensuales. Asimismo, siguiendo el método de Hayford y Malliaris (2004), el PER estimado es utilizado para calcular la prima implícita de las acciones (implicit equity premia) que conforman el IGBVL. Ambos indicadores de valorización del mercado de acciones, el PER y la prima implícita, proporcionan evidencia de que no habría existido una burbuja en el mercado de acciones peruano en el periodo analizado.

El trabajo se divide en una introducción y tres secciones. En la primera sección se discute sobre el concepto de burbuja de activos y sus posibles causas desde un punto de vista conceptual, dentro del cual se enmarcan los indicadores propuestos en el presente trabajo, tales como el ratio precio-utilidad de acciones y la prima por riesgo de la inversión en acciones. En la siguiente parte se desarrolla una metodología para calcular el PER agregado y la prima de riesgo agregadas del Índice General de la Bolsa de Valores de Lima (IGBVL); y se evalúa el desempeño de los precios del mercado de acciones peruano en el periodo 2001-2011. La última sección ofrece las conclusiones del trabajo.

\section{CONSIDERACIONES TEÓRICAS Y METODOLÓGICAS PARA EL CÁLCULO DEL RATIO PRECIO-UTILIDAD (PER)}

La mayoría de episodios de crecimiento sin precedentes de los precios de activos han estado asociados al concepto de burbuja. Este concepto, sin embargo, no está exento de controversia. El enfoque tradicional señala que una burbuja se presenta cuando el precio del activo se aparta significativa y crecientemente de su valor de equilibrio, arrastrado por elementos especulativos (Garber, 2000). Kindleberger (1978) define una burbuja como una sucesión de eventos vinculados: 
movimiento ascendente de precios a lo largo de un periodo extenso, seguido de una explosión y declinación de los mismos.

Es razonable pensar que bajas tasas de interés 4 pueden "alimentar" un crecimiento de precios de activos, sobretodo en un entorno de fuerte crecimiento del crédito y en las etapas de un ciclo económico alcista. Greenspan (2007) escribía en sus memorias:

La caída de las tasas reales de interés de largo plazo que ha ocurrido en las dos últimas décadas ha estado asociada con aumentos en los ratios precioutilidad de las acciones, inmuebles, y de hecho de todos los activos. (p. 14)

La relación inversa entre tasas de interés y precios de las acciones tiene sustento en el denominado Modelo de la Fed (FED Model). Fue Ed Yardeni, del Deutsche Morgan Grenfell, quien le dio el nombre de "Modelo de la Fed", basado en un reporte realizado por la Reserva Federal el 22 de julio de 1997 en el que se señala que

...cambios en este ratio (PER del S\&P 500) han estado inversamente relacionados a cambios en los rendimientos de largo plazo de bonos del Tesoro, pero este año los incrementos en los precios de las acciones no han sido seguidos por una disminución significativa en las tasas de interés. ${ }^{5}$

En efecto, en este reporte de 1997, la Fed afirma que las acciones estaban sobrevaloradas. La idea básica de este modelo es que en la medida en que bonos $\mathrm{y}$ acciones son activos sustitutos, sus rendimientos deben ser aproximadamente iguales en el largo plazo. Específicamente, el modelo compara el rendimiento de los bonos del tesoro estadounidense a 10 años con el rendimiento de las acciones medido como la inversa del ratio precio/utilidades del S\&P 500 de las utilidades proyectadas un año adelante. Si el rendimiento de las acciones (calculada como la inversa del PER) es mayor al de los bonos, entonces se concluye que las acciones

4 Que implican a su vez bajos rendimientos (y altos precios) de los bonos.

5 Sin embargo, se debe mencionar que la Reserva Federal nunca ha aceptado la existencia de este reporte. están subvaluadas o hay subinversión en acciones; si se presenta el caso contrario, se estima que las acciones están sobrevaloradas.

De la discusión anterior se puede señalar que el concepto de PER es uno de los indicadores más simples y difundidos para valorizar acciones. Este indicador se calcula de la siguiente manera:

$$
P E R=\frac{\text { Precio de la acción }}{\text { Utilidad por acción }}
$$

Es decir, el PER es un indicador que permite calcular el número de años en que se va a recuperar la inversión (representada por el precio pagado). Así, por ejemplo, si una acción tiene un PER de 10, significa que el precio pagado por dicha acción se recupera en 10 años dado el flujo de utilidades (esperado) de la empresa6.

La mayoría de países cuentan con índices de precios de acciones que son usados para seguir el desempeño de las principales plazas bursátiles mundiales. Entre los principales índices bursátiles tenemos al S\&P 500 (índice elaborado por la agencia calificadora Standard and Poor's) conformado por 500 empresas listadas mayoritariamente en la Bolsa de Valores de Nueva York 7. El Dow Jones Industrial (DJ) está compuesto por las 30 empresas industriales más importantes de Estados Unidos. También se tienen a DAX de Alemania; IPC de México; IPSA de Chile; IGBC de Colombia; Bovespa de Brasil; IGBVL e ISBVL de Lima, entre otros.

Desde el punto de vista práctico, usualmente se considera a los valores promedio históricos del PER como valores "normales" de determinado índice. Un PER por encima de su nivel "normal" o histórico es

6 En general, el PER será mayor según el riesgo o volatilidad de las utilidades de la empresa. Diferencias entre PER de acciones de diversos sectores pueden estar reflejando diferencias en riesgos (utilidades más volátiles) y no necesariamente diferencias en utilidades esperadas. Por ejemplo, las acciones de servicios públicos y de bebidas normalmente en promedio tienen un PER más bajo que las acciones tecnológicas o mineras debido a que la fuente de ingresos de las primeras son más estables.

7 El índice S\&P 500 está compuesto por lo siguiente: 400 empresas industriales, 40 de servicios públicos, 40 de instituciones financieras y 20 de empresas de transporte. El índice DAX está conformado por 30 acciones. 
una señal de una potencial sobrevaluación o desvío de las acciones de sus fundamentos. Shen (2000) utiliza el PER para analizar el episodio de aumento de los precios de la bolsa de valores de Nueva York (S\&P 500) antes del estallido de la burbuja Nasdaq. El PER histórico o normal es de mayor utilidad para "bolsas maduras"8 cuyas empresas son relativamente estables en el tiempo y los niveles de utilidades pasadas son una medida aceptable de sus utilidades futuras. De lo contrario, el valor histórico es solo referencial y debe evaluarse con indicadores de valoración alternativos.

$\mathrm{Al}$ igual que el PER, también se ha planteado el uso de la prima implícita de las acciones (implied equity premium) como indicador de sobrevaluación (Hayford \& Malliaris, 2004). El cálculo de la prima implícita en las acciones se deduce a partir de la ecuación de Gordon (Gordon, 1959 y 1962). El modelo de Gordon está dado por ${ }^{9}$ :

$$
P_{t}=\frac{D_{t}(1+g)}{i+\rho-g}
$$

donde $P_{t}$ es el precio de la acción; $D_{t}$ son los dividendos que paga la acción en el periodo; $g$ es la tasa de crecimiento de los dividendos que se asume constante, $i$ es la tasa de interés de descuento libre de riesgo, y $\rho$ es la prima por riesgo de la acción.

El modelo de Gordon establece que, en equilibrio, el precio de una acción es el valor de sus dividendos futuros (esperados) descontados mediante una tasa de descuento $(i+\rho)$ que toma en cuenta la tasa de interés libre de riesgo y una prima de tasa de interés que compense el riesgo de la acción (equity premia) (Benninga, 2001) ${ }^{10}$. Hayford y Malliaris y el FMI (2000) usan

8 "Bolsas maduras" se define como aquellas bolsas cuyas empresas son relativamente estables en el tiempo y los niveles de utilidades pasadas son una medida aceptable para sus utilidades futuras.

9 Esta derivación sigue de cerca al Fondo Monetario Internacional (2000), Capítulo 3.

10 El precio de una acción en el periodo actual, $P_{0}$, se puede expresar como el valor presente de los dividendos futuros, donde la tasa de interés de descuento es $r=i+\rho$ : (Benninga, 2001, 27-29).

$$
P_{0}=\sum_{t=1}^{00} \frac{D_{1}(1+g)^{t-1}}{(1+r)^{t}}=\frac{D_{1}}{r-g}, \text { siempre que } g<r
$$

como tasa libre de riesgo a la tasa de rendimiento del bono del Tesoro a 10 años. $\mathrm{Al}$ asumir este modelo una tasa de crecimiento constante de los dividendos, su utilidad es mayor, al igual que el PER, para la valorización de empresas maduras con dividendos estables o para índices de mercado agregados.

Si asumimos que los dividendos que paga una acción son (en el largo plazo) igual a sus utilidades, es decir $D=U$, entonces la ecuación (1) se puede expresar como:

$$
\frac{P_{t}}{U_{t}}=\frac{(1+g)}{i+\rho-g}
$$

De esta manera, el ratio precio-utilidad (PER) de "equilibrio" corresponde al valor presente de los dividendos (esperados) futuros. Un PER demasiado alto respecto a sus valores "normales" puede estar indicando un desvío del precio de la acción de sus fundamentos.

De la ecuación (2) resolvemos para la prima de interés implícita de la acción de largo plazo $\rho$ como:

$$
\rho_{t}=(1+g) \frac{U_{t}}{P_{t}}-i_{t}+g
$$

que a su vez se puede expresar como ${ }^{11}$ :

$$
\rho_{t}=\left(1+h_{t}\right)\left(1+\pi_{t}\right) \frac{U_{t}}{P_{t}}-i_{t}+h_{t}+\pi_{t}+h_{t} \pi_{t}
$$

donde: $\left(1+h_{t}\right)=\frac{\left(1+g_{t}\right)}{\left(1+\pi_{t}\right)}$

es la tasa de crecimiento real de los dividendos.

Para estimar la prima esperada para la inversión en acciones, Hayford y Malliaris y el FMI (2000) asumen que $h_{t}$ se puede aproximar en el largo plazo a la tasa de crecimiento del PBI potencial ${ }^{12}$, y aproximan la tasa nominal $i_{t}$ como la tasa del bono del gobierno a 10 años. El ratio $\frac{U_{t}}{P_{t}}$ es la inversa del PER del S\&P 500 (trailing) ${ }^{13}, \mathrm{y} \pi_{t}$ es la tasa de inflación.

11 Cfr. Hayford y Malliaris, p. 10, así como el informe del FMI (2000), Tabla 3.1, nota de página 4, p. 82.

12 FMI (2000) usa la tasa de crecimiento del PBI histórico.

13 Calculado sobre la base de las utilidades de los últimos cuatro trimestres. 
Para calcular la prima realizada (histórica), el FMI (2000) usa la tasa de crecimiento del PBI histórico. El crecimiento del PBI potencial se usa como "proxy" del crecimiento esperado de los dividendos.

Tanto Hayford y Malliaris como el FMI (2000) señalan que los altos ratios de $\mathrm{P} / \mathrm{U}$ que no corresponden a una retribución esperada adecuada para los inversionistas (prima por riesgo de invertir en acciones) es una señal de posible sobrevaloración de las acciones. Por el contrario, primas por riesgo (equity premia) por debajo de sus niveles "normales" o históricos pueden ser señal de burbuja.

Si se analiza el Modelo de la Fed en términos de la ecuación (2) del modelo de Gordon, este modelo sería el resultado de suponer que tanto el crecimiento de los dividendos $(g)$ como la prima por riesgo $(\rho)$ son iguales a cero. En ese caso, la ecuación (2) se interpreta de esta manera:

$$
\frac{P_{t}}{U_{t}}=\frac{\delta(1+g)}{i+\rho-g} \text { Modelo de Gordon }
$$

se convierte en: $\frac{U_{t}}{P_{t}}=i \quad$ Modelo de la Fed

El supuesto del Modelo de la Fed de una prima por riesgo igual a cero no es muy razonable, considerando las diferencias en el riesgo de las acciones y los bonos del Tesoro. En cambio, es razonable suponer que las acciones en el largo plazo deben tener un rendimiento superior a los bonos del Tesoro (una prima por riesgo positiva) que compense la mayor volatilidad (riesgo) de sus rendimientos.

Sin embargo, se debe considerar además que la evolución de la prima para la inversión en acciones está sujeta a diversos factores adicionales como por ejemplo oportunidades de diversificación de cartera, cambios en productividad de la economía, modificaciones en riesgo de los instrumentos, costos de transacción, impuestos, entre otros. En ese contexto, el crecimiento experimentado de los mercados bursátiles emergentes está explicado en parte por ganancias en productividad, menor riesgo y menores costos de transacción.

Jagannathan, McGrattan y Scherbina (2000) encuentra una caída significativa de la prima pagada por las acciones en Estados Unidos desde 7\% entre 1926-1970 a un nivel cercano a cero entre 1982 y 1999; esto último daría una base empírica al modelo de la Fed en dicho periodo. Sin embargo, De Long y Konstantin (2008) señalan que la prima para la inversión en acciones persiste y se espera sea positiva para el futuro; dicha prima se daría, no obstante, el menor riesgo de las inversiones en acciones por la mayor diversificación.

Shen (2000) y FMI (2000), antes de la caída de la bolsa de Estados Unidos en el 2001, encuentran ratios de PER para Estados Unidos bastante por encima de sus valores históricos. Asimismo, FMI (2000) encontró, antes de la caída bursátil del año 2000, valores de PER del S\&P 500 por encima de sus valores históricos (un promedio de 29.5 para 1999 frente a un promedio histórico de 15.6 para 1980-1999) y una prima por riesgo implícita de acciones para 1999 de $1.0 \%$ frente a un valor histórico de $2.8 \%{ }^{14}$.

\section{CÁLCULO DEL PER Y DE LA PRIMA POR RIESGO DE MERCADO PARA LA INVERSIÓN EN ACCIONES EN EL PERÚ}

Bloomberg, en su página (WPE), reporta estadísticas de la evolución de los principales índices bursátiles mundiales, así como de sus ratios precio /utilidad (PER) agregados que por lo general son reportados por las propias bolsas. En el caso del Perú, Bloomberg solo reporta el PER de acciones individuales dado que la Bolsa de Valores de Lima no publica el PER agregado de mercado de ninguno de sus índices ${ }^{15}$, a diferencia de las principales plazas bursátiles como el Dow Jones, Standard \& Poor's, Nasdaq, entre otros ${ }^{16}$, que sí lo hacen.

14 FMI (2000), Tabla 3.1. Para el periodo 1950-1999, el promedio del PER para S\&P 500 fue 15. Asimismo, Hayford y Malliaris reportan un promedio histórico de $8 \%$ para la prima implícita en el periodo 1960-1993.

15 Las acciones listadas en la BVL ascienden aproximadamente a 200. El número de acciones que componen los índices son variables en el tiempo, siendo en la actualidad el número de acciones del índice General (IGBVL) igual a 32, del Índice Selectivo (ISBVL) de 15, y del INCA de 20; la composición mayoritaria corresponde a empresas mineras. Cada semestre (el primer día útil de enero y julio), la BVL publica las ponderaciones vigentes de los respectivos índices, que están basadas en los montos negociados.

16 Hasta el 2006 Bloomberg publicó en su página una serie de PER para el IGBVL, la que empezaba en agosto de 
Con el fin de llenar el vacío existente, en el presente trabajo se estima una serie mensual del ratio precioutilidad (PER) del Índice General de la Bolsa de Valores de Lima (IGBVL) para el período enero 1995-octubre 2011. El ratio se obtiene de dividir la capitalización total de las empresas que componen el IGBVL entre la suma de las utilidades netas obtenidas por cada acción ${ }^{17}$ de dicho índice. Así,

$$
\text { PER IGBVL }=\frac{\text { Capitalización bursátil }}{\text { Utilidades totales }}
$$

donde: $P_{i}$ y $N_{i}$ corresponden a los precios y número de acciones de fin de período, respectivamente, de cada acción incluida en el IGBVL.

$U_{i}$ corresponde a las ganancias o utilidades por acción reportadas por la empresa en sus estados financieros de los últimos 12 meses (trailing).

$W_{i}$ es la utilidad de la acción i sobre las utilidades totales de las acciones del IGBVL.

El método de cálculo propuesto equivale a ponderar el PER individual de cada acción por su participación en el total de utilidades de las empresas que conforman el IGBVL (ver Anexo). Esta metodología de considerar las utilidades como ponderador presenta la menor volatilidad del PER agregado si se le compara con el método de ponderar los PER individuales por la capitalización bursátil o montos negociados ${ }^{18}$. Dicha volatilidad del PER se presenta cuando las empresas

2001. Esta serie presentaba valores inestables de los PER que fluctuaban entre 186 y 30 en el periodo junio 03-junio 2006. Bloomberg no reportó la metodología empleada para el cálculo del PER del IGBVL.

17 El IGBVL es un índice de precios (Laspeyres) que tiene como periodo base diciembre $1991=100$. La ponderación del IGBVL está dada por su negociación y está disponible desde noviembre del 1998 en: <www.bvl.com.pe>. Los datos de los PER y capitalización bursátil para cada acción se tomó de Economatica de los balances consolidados en dólares. Cuando no se cuenta con PER de balances consolidados, la información se tomó de balances no consolidados. Para el periodo 1995-1997, se tomó la composición del IGBVL de 1998. El número de acciones que componen el IGBVL desde julio 2011 es 32.

18 En el Anexo se presenta el cálculo del PER agregado con dichas ponderaciones alternativas. tienen PER negativos o bastante altos - debido a pérdidas o utilidades muy pequeñas - lo que dificulta el proceso de agregación. Para limitar el efecto de los PER negativos, algunos autores consideran la alternativa de excluir aquellas acciones con pérdidas, lo que se denomina non negative trailing PER ${ }^{19}$.

En la Figural se muestra el PER del IGBVL estimado a partir de la ecuación (7) para el período enero 95-octubre 2011. Se observa que, al mes de octubre del 2011, dicho ratio se ubica por debajo de su promedio histórico, que es igual a 17. Este cálculo del PER, al incorporar solo las utilidades de los últimos cuatro trimestres (trailing), no estaría recogiendo información de las utilidades "estables" o promedio de una empresa que son las relevantes cuando se toman decisiones de inversión. Shiller (2005) ${ }^{20}$ usa para el cálculo del PER al promedio de las utilidades anuales de los últimos 10 años, las que se ajustan por inflación, dando como resultado un PER real para las utilidades de los últimos 10 años, que denomina PER $10^{21}$.

Siguiendo a Shiller (2005), se calcula el PER real para el IGBVL usando el promedio de las utilidades anuales de los últimos 5 años (al que denominamos PER 5). El ratio del PER real calculado (PER 5) se muestra en la Figura 2, que tiene un promedio de 17 para el periodo analizado.

Con el fin de determinar si los valores observados del PER se encuentran dentro de sus valores normales, Bisciari, Durré y Nyssens (2003) proponen calcular una banda de confianza, sobre la base de los datos históricos $22, \mathrm{y}$ así determinar si existen indicios de sub o

19 También, existe la alternativa de considerar solo las ganancias operativas, lo que excluye ganancias que no corresponden al negocio principal de la empresa.

20 Shiller (2005) calcula el PER real ajustando las utilidades por acción del ciclo económico, usando para ello las utilidades por acción promedio de los últimos 10 años (CyclicallyAdjusted Price Earnings Ratio-CAPE).

21 Shiller (2005) usa el índice Standard \& Poor's de precios para el cálculo del PER y no el índice de retornos totales. El $44 \%$ de los retornos totales del segundo índice provienen de (reinversión de) dividendos en los últimos 80 años.

22 Bisciari, Durré y Nyssens (2003) analizan la series de precios y ganancias del Standard \& Poor's 500 para el período 1871-2002 estableciendo una banda de confianza respecto de la media. Los valores de la banda que se calculó para la 


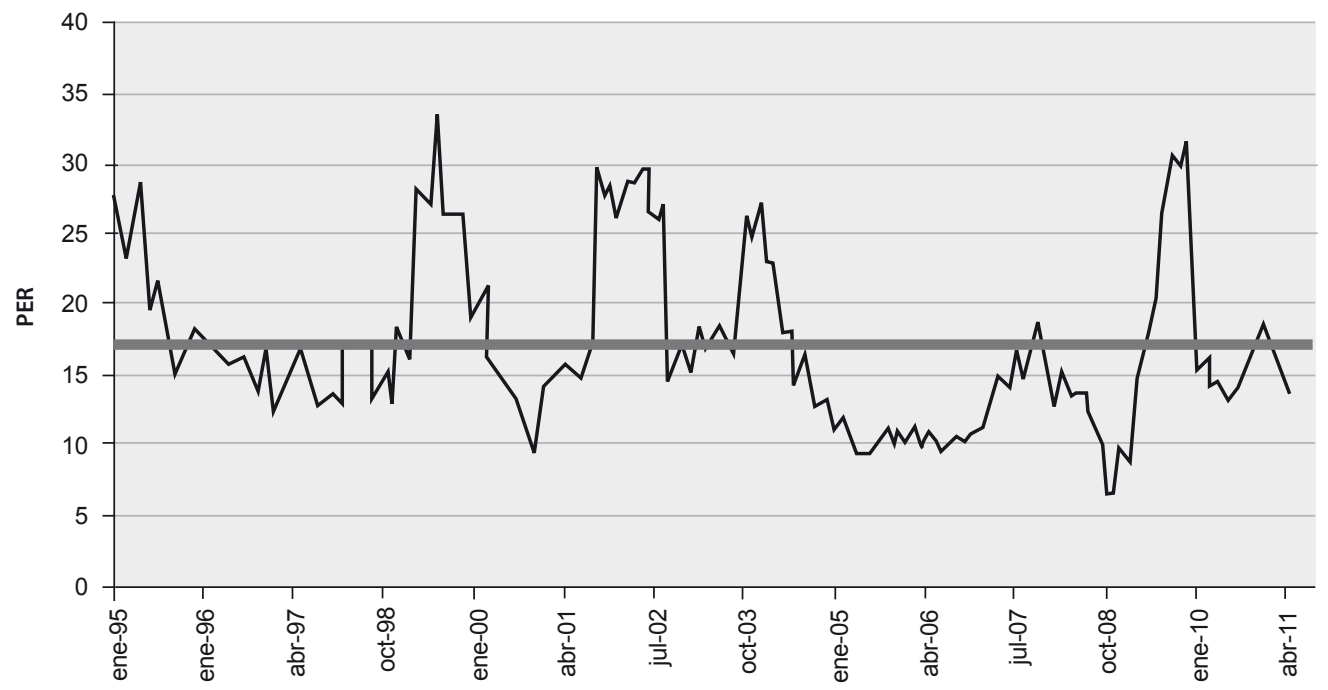

Fuente: Estimaciones del autor.

Figura 1. PER de Acciones del IGBVL: 1995 - 2011 (trailing)

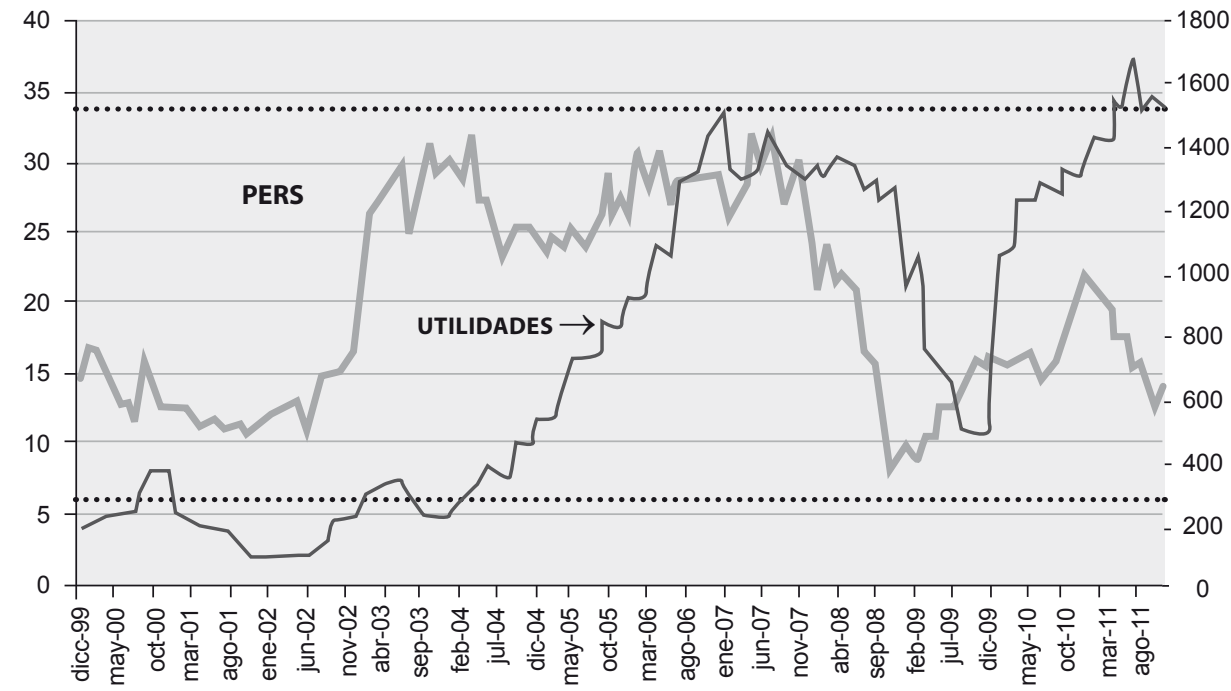

Fuente: Estimaciones del autor.

Figura 2. PER 5 e Índice de Utilidades del IGBVL: Dic. 1999 - Oct. 2011 (valores reales)

sobre valoración. Con base a los valores del PER 5 se obtienen las bandas al 95 por ciento de confianza con valores de 6.5 y 34 , que se muestran en la Figura 2 junto al índice de utilidades de las empresas 23 .

economía estadounidense fueron de 10 y 17,5 (una banda de $25 \%$ respecto de la media).
La evolución del PERS de la Figura 2 indicaría que no habría indicios de una burbuja en el periodo analizado, dado que este indicador se sitúa dentro de la banda de confianza estimada. Los altos niveles observados

23 Se asume una distribución normal de los rendimientos, con un intervalo de confianza de: $\left[\mu-{ }_{1,96} \sigma_{;} \mu+1,96 \sigma_{]}\right.$. 
para el PER 5 entre el 2003 hasta mediados del 2008 (por encima de la media del periodo) sería reflejo de expectativas optimistas sobre el crecimiento de la utilidades. Al respecto, desde fines del 2003 las utilidades registraron un crecimiento sustancial y se mantuvieron en niveles elevados hasta fines del 2008, producto de un crecimiento económico prolongado y de la evolución favorable de los precios de las materias primas (commodities). Es importante señalar que, aún en el periodo de mayor crecimiento de los precios de las acciones de la BVL (2006-2007) que precedió a la crisis del 2008, el PER 5 no indicaría la existencia de una sobrevaluación del mercado $d$ acciones.

El comportamiento del PER 5 se puede decir que anticipó la evolución de las utilidades de las empresas de la BVL. Así, el PER empieza a crecer fuertemente desde el 2003 anticipando el crecimiento de las utilidades que recién se produce hacia el 2004. Asimismo, el PER 5 comienza a descender rápidamente en el cuarto trimestre del 2007, mucho antes del desplome de las utilidades de las empresas que se produce hacia fines del 2008. En la actualidad, los niveles de PER son consistentes con el nivel de utilidades de las empresas, situándose incluso en un nivel menor a 15; esto es por debajo de su valor promedio del periodo analizado.

Siguiendo el procedimiento de Hayford y Malliaris descrito en la sección 2 de este trabajo, se calcula la prima por riesgo implícita y la prima por riesgo histórica de las acciones del IGBVL para el periodo enero 2001-setiembre 2011 (ver Figura 3). Se observa que la prima implícita esperada por los inversionistas (línea sólida), cuyo promedio es de alrededor $7.5 \%{ }^{24}$, tiene una tendencia creciente desde el 2004 hasta el tercer trimestre del 2008, coincidiendo con la evolución registrada por el PER 5. Ello abonaría a favor de la hipótesis de que el precio de las acciones respondió a expectativas justificadas de ganancias de las empresas en el periodo de auge bursátil25. Asimismo, en la actualidad se observan primas implícitas positivas y muy

24 La prima esperada para el periodo 2005 -set. 11 es de $11.2 \%$ y la prima histórica es de $11.9 \%$ para el mismo periodo.

25 La prima del rendimiento de las acciones del IGBVL (en exceso del rendimiento de los bonos) es en promedio positiva en el periodo analizado, con la excepción del periodo febrero 2001-agosto 2002. superiores al promedio histórico, lo que indicaría que no existiría evidencia de sobrevaloración del mercado de acciones peruano.

Si como señala Shiller (2005) una burbuja surge cuando los precios de los activos reflejan expectativas de escenarios demasiado optimistas y altamente improbables, lo que denomina "exuberancia irracional", es poco probable que la evolución de la prima por riesgo analizada y los precios de las acciones de la bolsa peruana en el periodo analizado hayan tenido elementos de burbuja. A diferencia de una burbuja, que está basada en un alto porcentaje en expectativas no validadas, el alza registrada en el mercado de acciones peruano estuvo explicada por expectativas positivas de mayores utilidades basada en fundamentos como un elevado crecimiento económico y altos precios de los minerales ${ }^{26}$. La corrección de precios de las acciones que se dio a fines del 2008 y durante el 2009 respondió al deterioro de las variables fundamentales. Sin embargo, a partir de los dos últimos años los precios de las acciones en el mercado peruano se han recuperado debido a la mejora en las perspectivas de las utilidades de las empresas 27 que se incorporan en el aumento que viene registrando la prima esperada 28 .

26 El caso de Estados Unidos es distinto. Como en la crisis de 1929, durante la mitad del 2008 los precios de las acciones en la bolsa norteamericana continuaban al alza a pesar de que la economía norteamericana ya se encontraba en recesión, aunque esto fuera anunciado oficialmente recién en octubre del 2008.

27 Durante el "boom" del mercado de acciones del 2006-2007 se habló de "superciclo". Según esta teoría, un superciclo es un periodo histórico de elevado crecimiento global con una duración de una o dos generaciones, no exento de volatilidades. Actualmente, estaríamos en un tercer superciclo que comenzó en el 2000. El primero se dio entre 1870 y 1913 que determinó la emergencia de Estados Unidos, el segundo duró entre 1946 y 1973 y vislumbró la emergencia de Japón y los tigres asiáticos. El tercero estaría marcando el liderazgo de China e India, y también de algunos países emergentes de América Latina. Para esto consultar Motianey (2010) y Standard Chartered (2010).

28 La existencia de elevados niveles de prima de las acciones peruanas (en promedio 7.5\% para el periodo 2001-2011) es un elemento positivo que puede atraer a potenciales inversionistas del exterior y ayudar así a dinamizar la bolsa de valores peruana. 


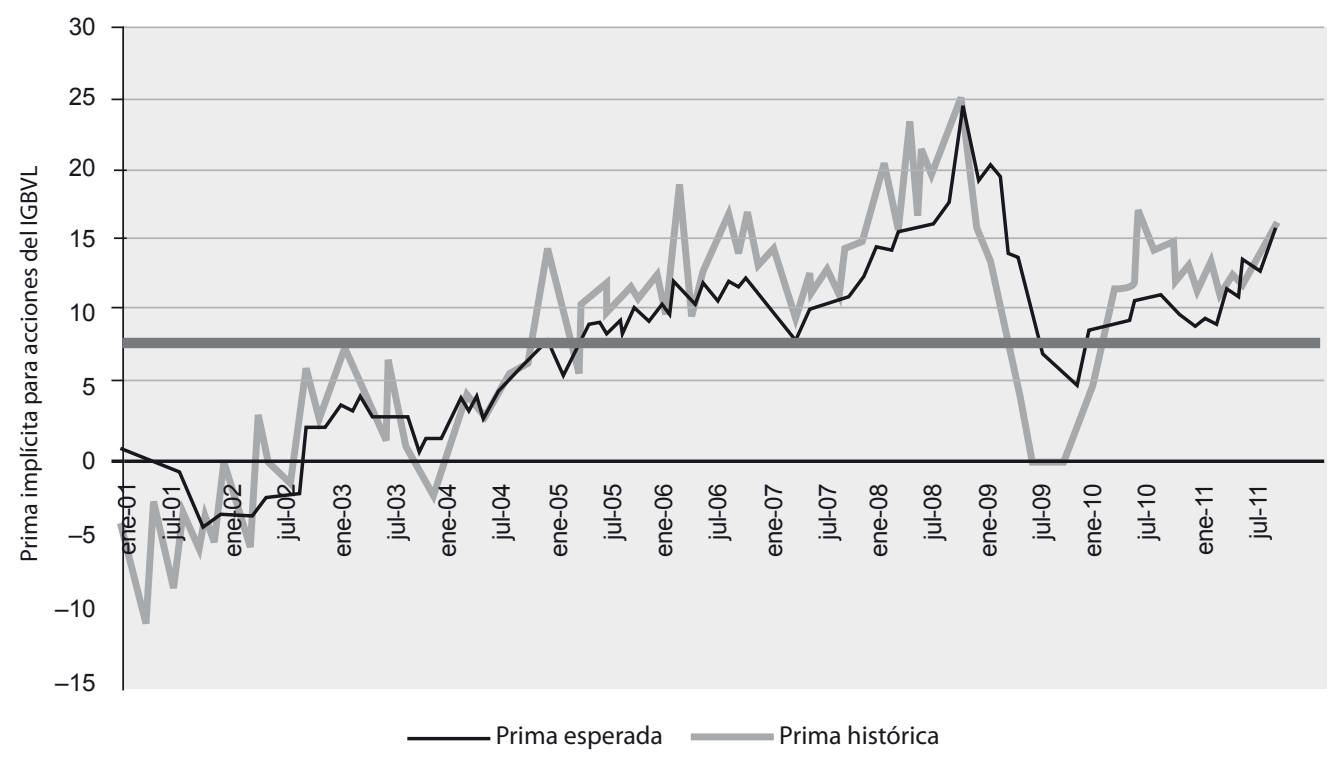

Fuente: Estimaciones del autor.

Figura3. Prima implícita para acciones del IGBVL: esperada e histórica (porcentaje anual)

\section{RESUMEN Y CONCLUSIONES}

La crisis financiera actual tiene su origen en la crisis hipotecaria iniciada en el período 2007-2008 en los Estados Unidos. Los altos precios de las viviendas fueron impulsadas por un crecimiento sustancial del crédito, en un entorno de bajas tasas de interés y expectativas optimistas sobre la economía. Los efectos de la crisis afectaron la marcha de la economía generando un proceso recesivo en los Estados Unidos que afectó, en mayor o menor medida, a la mayoría de las economías mundiales, incluido el Perú.

En tanto los precios de activos puedan tener efectos sobre la economía es importante contar con indicadores que permitan evaluar la existencia de posible alejamiento de estos de sus fundamentos. En el presente trabajo se desarrolla una metodología para calcular el ratio precioutilidad (PER) para el Índice General de la Bolsa de Valores de Lima (IGBVL), el que se ajusta siguiendo el método propuesto por Shiller (2005). A partir del PER calculado, siguiendo a Hayford y Malliaris, se estima la prima implícita esperada por los inversionistas de la inversión en acciones.

Los resultados de la estimación del PER como de la prima implícita para el periodo 1995-2011 no muestran indicios de la existencia de una burbuja en el mercado de acciones. Se observa que el precio de las acciones (PER 5) respondió básicamente a la evolución esperada de las utilidades de las empresas (prima implícita), no observándose elementos de sobrevaluación incluso durante el periodo de auge de la bolsa de valores que precedió a la crisis financiera externa de 2008.

En este sentido, la existencia de una prima implícita positiva de los rendimientos del mercado de acciones peruano se torna atractiva para potenciales inversionistas del exterior. Ello ayudaría a dinamizar el mercado de valores doméstico, aumentando su liquidez y profundidad como viene ocurriendo con el mercado de bonos peruano en los últimos años y que está atrayendo una creciente participación de inversionistas no residentes. 


\section{ANEXO}

\section{Metodologías Alternativas para Calcular el PER del IGBVL}

\section{A. Ponderación basada en las utilidades}

Para una acción se cumple que:

$$
P E R=\frac{\text { Capitalización bursátil de la acción }}{\text { Utilidades totales de la acción }}=\frac{P N}{U}=\frac{P}{\frac{U}{N}}=\frac{\text { Precio }}{\text { Utilidad por acción }}
$$

Para las acciones del IGBVL:

$$
\text { PER IGBVL }=\frac{\sum P_{i} N_{i}}{\sum U_{i}}=\sum \frac{P_{i} N_{i} U_{i}}{U_{i} \Sigma U_{i}}=\sum P E R_{i} \frac{U_{i}}{\Sigma U_{i}}=\sum P E R_{i} W_{i}
$$

donde: $\quad w_{i}=\frac{\text { Utilidades de la acción }}{\text { Utilidades totales del IGBVL }}$

\section{B. Ponderación basada en la capitalización bursátil}

$$
\text { PERIGBVL }=\sum P E R_{i} w_{i}
$$

donde: $w_{i}=\frac{\text { Capitalización de la acción }}{\text { Capitalización total del IGBVL }}$

\section{Ponderación basada en la ponderación semestral del IGBVL publicada por la}

\section{Bolsa de Valores de Lima}

$$
\text { PER IGBVL }=\sum P E R_{i} w_{i}
$$

donde: $w_{i}=$ Ponderación de cada acción en el IGBVL

En la Figura 4 se presentan las tres alternativas de cálculo del PER para el periodo enero 1995-octubre 2011 con las ponderaciones alternativas arriba mencionadas. Se observa que la alternativa de usar las utilidades de cada empresa como ponderador da como resultado un PER agregado con menor volatilidad (una desviación estándar de 5.9), mientras que las otras dos alternativas generan PER con valores extremos tanto positivos como negativos. 


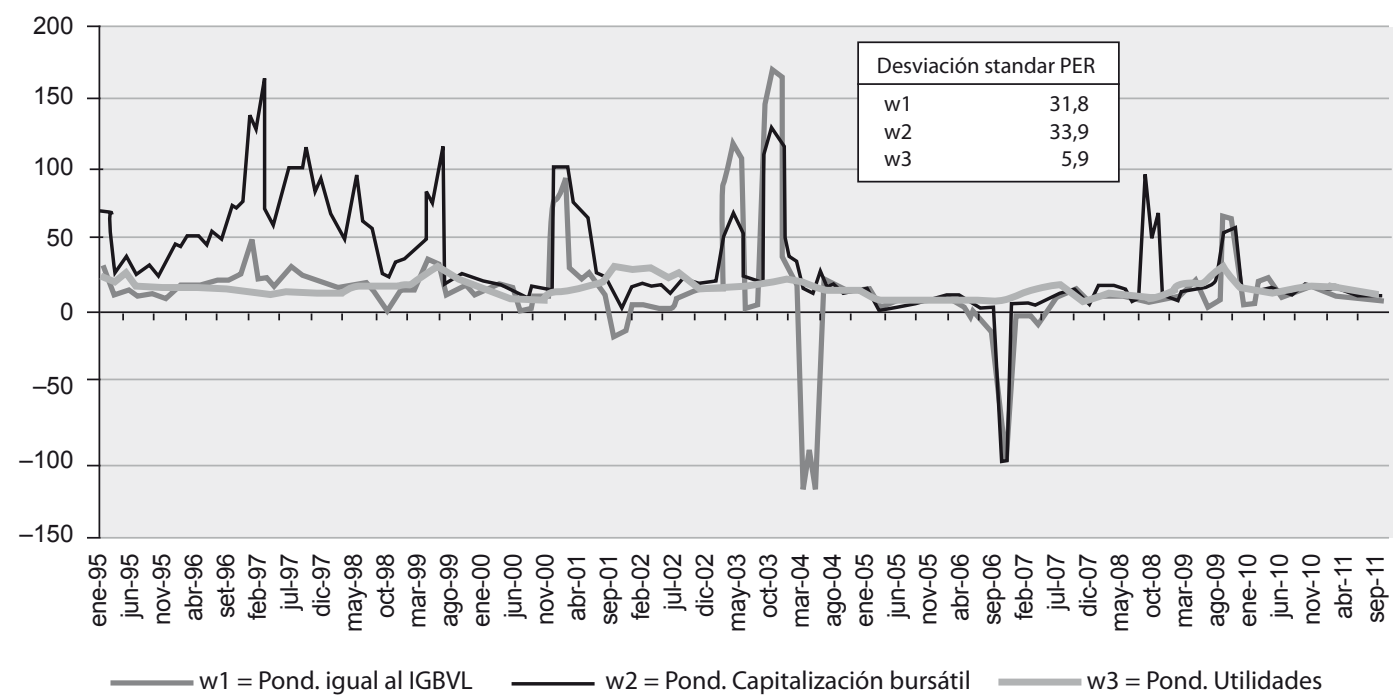

Fuente: Estimaciones del autor.

Figura 4. PER alternativos con diferentes ponderaciones

\section{Referencias}

Benninga, S. (2001). Financial Modeling (2nd Ed.). Cambridge, MA: The MIT Press.

Carmassi, J., Gross, D., \& Micossi, S. (2009). The Global Financial Crisis: Causes and Cures. Journal of Common Studies, 47(5), 977-996.

De Long, B., \& Konstantin, M. (February, 2008). The U.S. Equity Return Premium: Past, Present and Future (Mimeo). University of California at Berkeley.

Fondo Monetario Internacional (May, 2000). Asset Prices and the Business Cycle. World Economic Outlook. Occasional Paper Series.

Garber,P. (2000). Famous First Bubbles: The Fundamentals of Early Manias. Cambridge, MA: The MIT Press.

Gordon, M. J. (1959). Dividends, Earnings and Stock Prices. Review of Economics and Statistics, 41, 99-105.

Gordon, M. J. (1962). The Investment, Financing and Valuation of the Corporation. Homewood, ILL: Irwin.
Greenspan, A. (2007) The Age of Turbulence: Adventures in a New World. New York: Pengüin Press.

Hayford, M. D., \& Malliaris, A. G. (2004). Monetary Policy and the U.S. Stock Market. Economic Inquiry,42(3), 387-401.

Jagannathan, R., McGrattan, E., \& Scherbina, A. (2000). The Declining U.S. Equity Premium. Federal Reserve Bank of Minneapolis Quarterly Review, 24(4), 3-19.

Kindleberger, Charles (1978), Manias, panics, and crashes. New York, Basic Books.

Motianey, A. (2010). Super Cycles. The new economic force transforming global markets. New York: McGraw Hill.

Salas, J., \& y Ferreyra, J. (2006). Tipo de cambio real de equilibrio en el Perú: modelos BEER y construcción de bandas de confianza. (Documento de Trabajo BCRP N 006-2006). Lima, Perú: Banco Central de Reserva. 
Shen, P. (2000). The P/E Ratio and Stock Market Performance. Economic Review Federal Reserve Bank of Kansas City, Fourth Quarter, 23-36.

Shiller, R. J. (1999). Measuring Bubble Expectations \& Investor Confidence. (NBER Working Paper $\mathrm{N}^{\circ}$ 7008). Cambridge, MA: NBER.

Shiller, R. J. (2005). Irrational Exuberance. (2nd Ed.). Oxford: Princeton University Press.

Standard Chartered (2010). The Super-Cycle Report. Retrieved from <ttps://research.standardchartered. com/researchdocuments/Pages/ResearchArticle. aspx?\&R=73895>.
Summers, L. (2011). Why the Housing Burden Stalls America's Economic Recovery, Financial Times, 23.

Yardeni, E. (1997). Fed's Stock Market Model Finds Overvaluation. US Equity Research. Frankfurt, Hesse, Germany: Deutsche Bank.

Winkelried, D., \& Rodríguez, D. (2011). ¿Qué explica la evolución del tipo de cambio real de equilibrio en el Perú? 1992-2009. Revista Moneda, 147, 9-14. 